\title{
Paclitaxel, Iphospamide, and Cisplatin (TIP) as Bleomycin, Etoposide, and Cisplatin (BEP) Alternative for First-Line Therapy of Metastatic Germ Cell Tumor (GCT): A Case Series
}

\author{
Syamsu Hudaya, Adianti Khadijah \\ Department of Surgery, Fatmawati General Hospital, Jakarta, Indonesia
}

\section{ARTICLE INFO}

Received : 24 June 2019

Reviewed : 28 June 2019

Accepted : 27 September 2019

\section{Keywords:}

metastatic germ cell tumor, chemotherapy, testicular cancer

\author{
*Corresponding author: \\ Syamsu Hudaya \\ Department of Surgery, Fatmawati \\ General Hospital, Jakarta, Indonesia \\ shudaya.15@gmail.com
}

\begin{abstract}
A BSTRACT
Background: Bleomycin, etoposide, and cisplatin (BEP) is a standard first-line therapy for metastatic germ cell tumor (GCT), while paclitaxel, ifosfamide, and cisplatin (TIP) are commonly used as salvage therapy after failed BEP treatment. The unavailability of first-line drugs can be the reason for the use of second-line therapy. In this paper, we reviewed two initial cases of patients with metastatic GCT treated with TIP as first-line chemotherapy in our center.
\end{abstract}

Case Presentation: We reviewed the medical record and followed up two patients who had been treated with TIP as first-line chemotherapy for metastatic GCT due to lack of BEP regiment. We evaluated efficacy and toxicity of this treatment. These two patients were diagnosed with seminoma, with intermediate-risk according to International Germ Cell Cancer Collaborative Group (IGCCCG) classification. Both achieved complete response after four courses of TIP chemotherapy with toxicities mainly consisted of myelosuppression.

Conclusions: TIP demonstrated efficacy serves as the first-line therapy for germ cell tumors with an acceptable safety profile. Further studies with larger subjects are still needed for evaluation. However, TIP is more expensive compared to BEP, making BEP is still superior to TIP in public hospital setting where cost-effectiveness of treatment is important.

\section{INTRODUCTION}

Germ cell tumor (GCT) is a relatively rare disease, accounting for only $1 \%$ of all malignancies in men $[1,2]$. However, it still represents the most common solid tumor in men between 15 and 35 years of age [3]. There has been a marked increase in the incidence of testicular cancer worldwide [4]. In contrast to the incidence, there has been a remarkable decline in testicular cancer mortality over the past 30 years. Untreated testicular cancer may metastasize and eventually lead to death, but advances in treatment have resulted in increases in 5 -year survival rates from $63 \%$ to more than $90 \%$ during the last 3 decades $[5,6]$. The introduction of cisplatin-based chemotherapy regimens has resulted in the conversion of metastatic GCTs with an extremely poor prognosis into a curable solid malignancy. Among several regimes available, BEP, which consists of bleomycin, etoposide, and cisplatin, has proved to be highly efficacious against metastatic GCTs and is currently the most widely used regimen as the first-line therapy for metastatic GCTs [7].

However, the likelihood of sensitivity to chemotherapy and cure varies significantly on the basis of clinical and pathologic factors, which have been incorporated into the International Germ Cell Cancer Collaborative Group (IGCCCG) prognostic model. Although good-risk patients in this model have an approximately $90 \%$ progressionfree survival (PFS) rate after chemotherapy, the corresponding rates for intermediate-risk and poor-risk patients are only $70 \%$ to $75 \%$ and $45 \%$ to $55 \%$, respectively [8].

The combination of paclitaxel, ifosfamide, and cisplatin (TIP) was previously evaluated as a second-line treatment in phase I/II study of patients with testicular primary GCTs who relapsed after a favorable response to first-line chemotherapy. This regiment is also currently evaluated as the first-line therapy for metastatic GCT with intermediate and poor risks according to IGCCCG classification with satisfying results [9]. The unavailability of first-line drugs can be the reason for the use of second-line therapy as we know delayed management of metastatic cancer will increase progressivity and decrease overall survival.

At our institution, BEP was also the first-line chemotherapy to treat patients with metastatic GCTs until recent events where etoposide as the main component isn't available at our center due to several reasons. As a replacement, we treated patients with metastatic GCTs with TIP regiment based on several 
previous studies. In this paper, we reviewed two initial cases of patients with metastatic GCT treated with TIP regiment as first-line therapy in our center due to lack of $\mathrm{BEP}$ regiment.

\section{CASE PRESENTATION}

During the last six months, there have been 2 patients with metastatic GCTs treated with TIP. We reviewed each case in this paper. We followed up each case during chemotherapy and evaluated the chemotherapy response and toxicity to each patient.

The TIP regiment consisted of paclitaxel $250 \mathrm{mg} / \mathrm{m} 2$ by $24-\mathrm{h}$ infusion on day 1 , followed by ifosfamide 1.5 $\mathrm{g} / \mathrm{m} 2$ infusion over 2 hours and cisplatin $25 \mathrm{mg} / \mathrm{m} 2$ given over 2 hours on days 2-5. The dosages and schedule for cisplatin and ifosfamide administration were identical to the TIP regimen reported by The National Comprehensive Cancer Network (NCCN) Guidelines on testicular cancer. Mesna $500 \mathrm{mg} / \mathrm{m} 2$ was administered intravenously before ifosfamide infusions and every 4 hours for a total of three doses per day Courses were repeated every 21 days. ${ }^{10}$ All patients received prophylactic premedication with $20 \mathrm{mg}$ dexamethasone 12 and 6 hours before paclitaxel and intravenous ranitidine and oral diphenhydramine (each $50 \mathrm{mg}$ ) 30 min prior to paclitaxel administrations, as a standard chemo protocol in our hospital. Standard anti-emetic and hydration protocols were followed.

Table 1. TIP chemotherapy regimen used in this case series compared to standard BEP regimen

\begin{tabular}{ll}
\hline \multicolumn{1}{c}{ TIP course } & \multicolumn{1}{c}{ BEP course } \\
\hline $\begin{array}{ll}\text { Paclitaxel } 250 \mathrm{mg} / \mathrm{m} 2 \mathrm{IV} \text { on day-1 } \\
\text { Ifosfamide } 1500 \mathrm{mg} / \mathrm{m} 2 \text { IV on }\end{array}$ & $\begin{array}{l}\text { Etoposide } 100 \mathrm{mg} / \mathrm{m} 2 \\
\text { IV on days } 1-5\end{array}$ \\
$\begin{array}{l}\text { days } 2-5 \\
\text { Mesna } 500 \mathrm{mg} / \mathrm{m} 2 \text { IV before }\end{array}$ & IV on days $1-5$ \\
ifosfamide, and then 4 and 8 & Bleomycin 30 Units \\
hours after each ifosfamide dose & IV weekly on Days \\
on days $2-5$ & 1,8 , and 15 \\
Cisplatin $25 \mathrm{mg} / \mathrm{m} 2$ IV on days $2-5$ & Repeat every 21 \\
Repeat every 21 days & days \\
\hline
\end{tabular}

We evaluated chemotherapy response with the blood tumor marker and computed tomography (CT) scan following the complete course of chemotherapy. A complete response (CR) to chemotherapy alone was defined as marker normalization and radiographic resolution or marker normalization plus surgery revealing only necrosis or teratoma (no viable GCT). A CR to chemotherapy plus surgery is defined as marker normalization and complete surgical resection revealing viable GCT with negative margins. A partial response with negative tumor markers (PR-negative) is defined as marker normalization with residual radiographic abnormalities but without progression. The CR and PRnegative were considered favorable responses and required confirmation at 4 weeks or later by tumor markers and chest x-ray. An incomplete response (IR) was anything other than CR or PR-negative [11].

Toxicities were graded according to the National Cancer Institute Common Terminology Criteria for Adverse Events, version 4.0. Grade refers to the severity of the adverse events based on the general guideline as follows in Table 2 [12]. After at least one dose of chemotherapy, the patient was eligible for toxicity assessment.

Table 2. Grading of chemotherapy adverse events according to National Cancer Institute Common Terminology Criteria for Adverse Events, version 4.0.

\begin{tabular}{ll}
\hline Grade $\quad$ Description \\
\hline
\end{tabular}

1 Mild; asymptomatic or mild symptoms; clinical or diagnostic observations only; intervention not indicated

2 Moderate; minimal, local or noninvasive intervention indicated; limiting the ageappropriate instrumental activity of daily living

3 Severe or medically significant but not immediately life-threatening; hospitalization or prolongation of hospitalization indicated; disabling; limiting self-care activity of daily living.

$4 \quad$ Life-threatening consequences; urgent intervention indicated.

5 Death related to adverse events.

\section{Case 1}

The first case was a forty-one-year-old male, with the primary tumor on the left testicle. Preoperatively, the primary tumor was already spread to multiple lymph nodes in his left inguinal and paraaortic region. From pathological examination after left orchidectomy, it was testicular seminoma. After surgery, LDH and B-hCG were still elevated. He underwent TIP regiments for 4 cycles during 4 months. After the first chemotherapy cycle, the patient was admitted to the hospital with the low levels of hemoglobin $(5.6 \mathrm{~g} / \mathrm{dL})$, leucocyte $(1,200 / \mathrm{ul})$, and thrombocyte $(57,000 / \mathrm{ul})$, each of which needed transfusion (Grade 3 ). This patient had been treated in the hospital for 2 weeks. Cycles 2 to 4 of chemotherapy passed without severe adverse events, and upon follow 
up after courses he had complete chemotherapy response, with all tumor marker level normalized and no lymph node enlargement in follow up CT (Figure 1). Another nonhematologic adverse event experienced by this patient included nausea and vomitus (Grade 2).

\section{Case 2}

A forty-three-year-old male came with intraabdominal tumor. Upon history taking and physical examination, we found out that he didn't have intrascrotal left testicle since birth. Intraoperatively, the intraabdominal mass was removed. However, there were still parts of the tumor that can't be dissected. Pathological examination confirmed that the intraabdominal tumor was actually a GCT; seminoma, specifically. This patient underwent four cycles of TIP chemotherapy due to residual mass, paraaortic and inguinal lymph node enlargement, and elevated LDH and B-hCG. During the third chemotherapy cycle, the patient experienced anemia $(7.7 \mathrm{~g} / \mathrm{dL})$ and thrombocytopenia $(89,000 / \mathrm{ul})$, both requiring transfusion (Grade 3). This adverse event didn't happen in the first, second, and last chemotherapy cycles. We reassessed the patient following treatment, and both tumor markers had already been at the normal level and no apparent tumor mass from the CT (Figure 2).

\section{DISCUSSION}

Standard first-line chemotherapy regimens of GCT consist of BEP or without bleomycin (EP). However, the likelihood of sensitivity to chemotherapy and cure varies significantly on the basis of clinical and pathologic factors. According to IGCCCG prognostic model, goodrisk patients have an approximately $90 \%$ PFS rate after chemotherapy, the corresponding rates for intermediaterisk and poor-risk patients are only $70 \%$ to $75 \%$ and $45 \%$ to $55 \%$, respectively [9].

Meanwhile, the TIP regiment was originally developed as first-line salvage chemotherapy for testicular germ cell cancer patients who relapsed after the complete response (CR) or partial response (PR) with tumor marker-negative findings (PRm -) to prior chemotherapy $[13,14]$. As for TIP therapy following BEP failure as 2nd line therapy, Mead et al. [16] reported that the response rate was $38 \%$ and long-term disease-free survival was shown in $60 \%$ of cases.

In this paper, we reviewed TIP efficacy and toxicity as first-line chemotherapy for metastatic germ cell tumors as lack of BEP regimen in our center, especially for etoposide. Both of our cases were seminoma with intermediate-risk according to IGCCCG classification and both cases achieved complete response with chemotherapy alone. This result in line with Feldman et al, which reported $61 \%$ complete response for the intermediate-risk group, and $66 \%$ for the poor-risk group in response of TIP regiment as first-line therapy for germ cell tumor. Feldman et al also found that $94 \%$ patients with intermediate-risk achieve favorable response, compared to $74 \%$ patients of the poor-risk group [11].

Toxicities of TIP consisted mainly of myelosuppression and sensory neuropathy [15-17]. Both patients in this paper experienced Grade 3 of either anemia, leukocytopenia, or thrombocytopenia requiring a blood transfusion, support with granulocyte colony-stimulating factor and treated as inpatient. Nonhematologic toxicities experienced by these two patients are nausea and vomiting. No other treatment side effect reported, and more importantly, no death-related with treatment reported.

According to these two cases, TIP is a safe and effective first-line therapy for metastatic germ cell tumors. However, we assessed both treatment costs and it appears that with the same duration and course of treatment, TIP were roughly seven times more expensive than BEP. With a national healthcare system currently applied at our hospital, more expensive treatment will be more difficult to insurance covered, thus it would still be more convenient to use BEP as a primary treatment.

\section{CONCLUSIONS}

TIP demonstrated efficacy as first-line therapy for germ cell tumors with an acceptable safety profile as an alternative to standard BEP regiment. Further study with a larger sample is needed to evaluate this, and if needed, compare TIP with BEP head to head in term of efficacy and tolerability. However, TIP is far more expensive compared to BEP, making BEP is still superior to TIP in hospital setting where cost-effectiveness of treatment is important.

\section{DECLARATIONS}

\section{Competing of Interest}

There is no conflict of interest in this study.

\section{Acknowledgment}

No acknowledgment statement for this study 

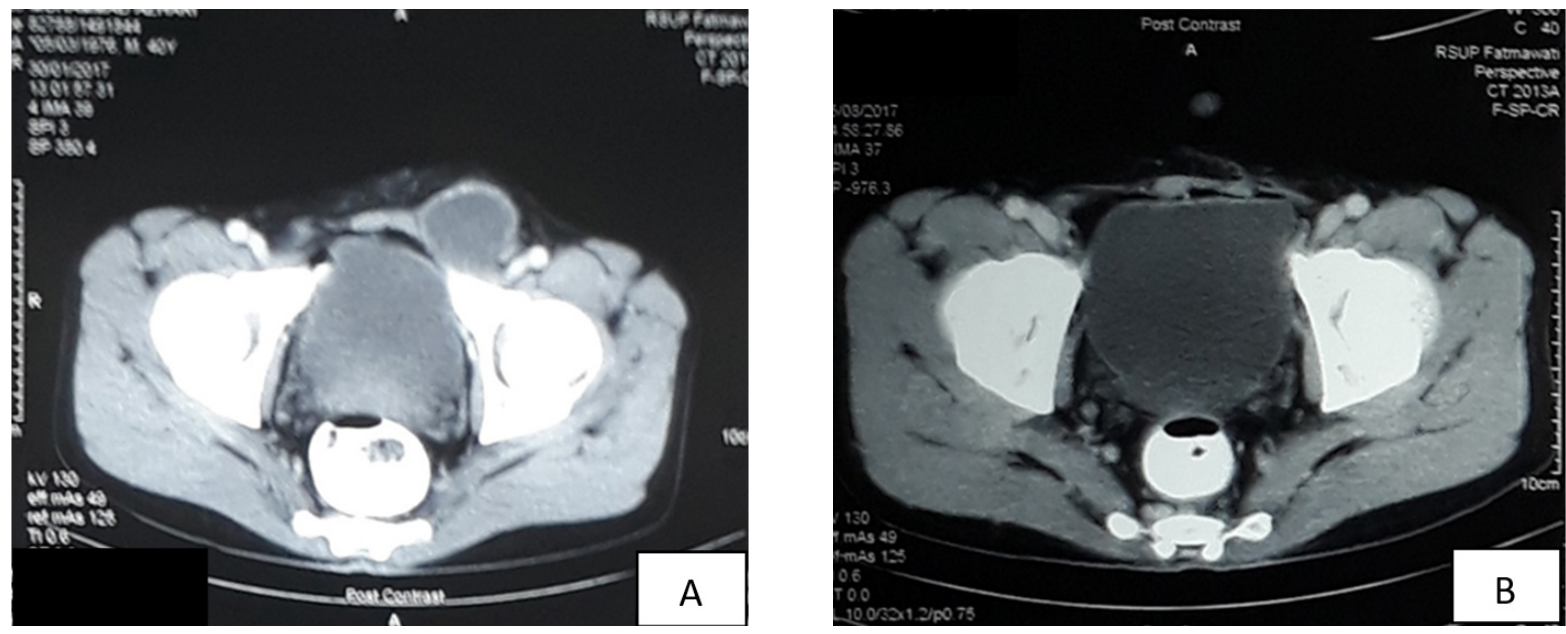

Figure 1. Left inguinal mass before (a) and after (b) four-course of chemotherapy.
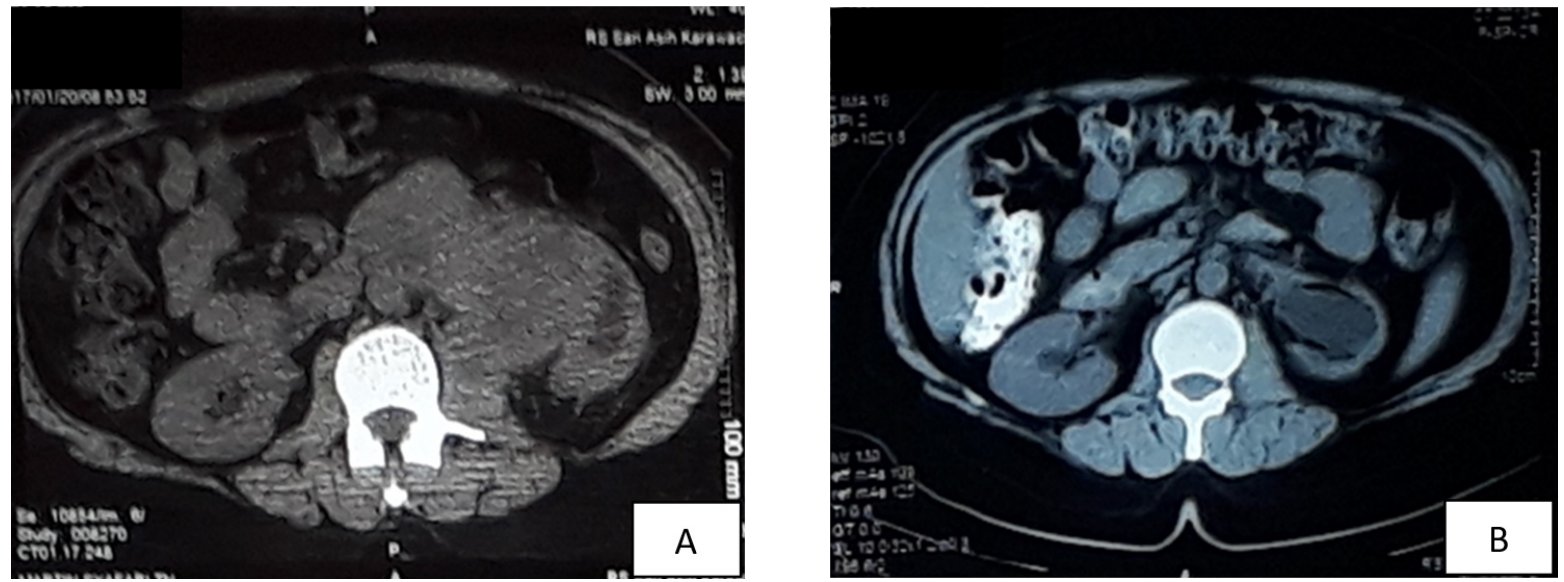

Figure 2. Intraabdominal mass before (A) and after (B) four-course of chemotherapy

Table 3. Summary of two cases

\begin{tabular}{lll}
\hline \multicolumn{1}{c}{ Characteristics } & \multicolumn{1}{c}{ Case 1} & \multicolumn{1}{c}{ Case 2} \\
\hline Age & 41 yo & 43 yo \\
Primary site & Left testicle & Intraabdominal (undescended testicle) \\
Pathology & Seminoma & Seminoma \\
AFP before chemotherapy & $2.7(\mathrm{~N})$ & $3.5(\mathrm{~N})$ \\
B-hCG before chemotherapy & $94(\uparrow)$ & $14.8(\uparrow)$ \\
LDH before chemotherapy & $1968(\uparrow)$ & $623(\uparrow)$ \\
$\begin{array}{l}\text { CT scan features before } \\
\text { chemotherapy }\end{array}$ & Dense mass on the left scrotal area expanding to & Multiple lymphadenopathies on the left \\
IGCCCG Classification & left inguinal, multiple paraaortal lymphadenopathy & inguinal, parailiac, and paraaortal region \\
Number of cycles & Intermediate risk & Intermediate risk \\
Adverse events & 4 & 4 \\
AFP after chemotherapy & $12.7(\mathrm{~N})$ & Anemia, thrombocytopenia \\
B-hCG after chemotherapy & $2.5(\mathrm{~N})$ & $6.4(\mathrm{~N})$ \\
LDH after chemotherapy & $239(\mathrm{~N})$ & $<1.2(\mathrm{~N})$ \\
CT scan features after & Shrinkage of paraaortal lymph node \\
chemotherapy & $($ size $<0.5 \mathrm{~cm})$, no visible tumor mass & $273(\mathrm{~N})$ \\
\hline
\end{tabular}

*AFP, alpha-fetoprotein; LDH, lactate dehydrogenase; B-hCG, beta-human chorionic gonadotropin; IGCCCG, International Germ Cell Cancer Collaborative Group; $\mathrm{CT}$, computed tomography 


\section{REFERENCES}

1. Parkin DM, Bray F, Ferlay J, Pisani P. Global cancer statistics, 2002. CA Cancer J Clin. 2005;55: 74-108.

2. Ferlay J, Bray F, Pisani P. GLOBOCAN 2000: cancer incidence, mortality, and prevalence worldwide. IARC Cancer Base No, 5. Lyon: IARC: 2001.

3. Schmoll HJ, Souchon R, Krege S, Albers P, Beyer J, Kollmannsberger $C$, et al. European consensus on diagnosis and treatment of germ cell cancer: a report of the European Germ Cell Cancer Consensus Group (EGCCCG). Ann Oncol. 2004;15: 1377-99.

4. Shanmugalingam $T$, Soultati $A$, Chowdhury $S$, Van Hemelrijck M. Global incidence and outcome of testicular cancer. Clin Epidemiol. 2013;5: 417-427.

5. Bray F, Richiardi L, Ekborn A, Pukkala E, Cuninkova $\mathrm{M}$, Moller $\mathrm{H}$. Trends in testicular cancer incidence and mortality in 22 European countries: continuing increases in incidence and declines in mortality. Int J Cancer. 2006;118(12): 3099-111.

6. Ehrlich Y, Margel D, Lubin MA, Baniel J, Advances in the treatment of testicular cancer. Transl Androl Urol. 2015;4(3): 381-90.

7. Einhorn LH and Donohue JP: Advanced testicular cancer: update for urologists. J Urol. 1998:160: 1964-9.

8. International Germ Cell Cancer Collaborative Group: International Germ Cell Consensus Classification: A prognostic factor-based staging system for metastatic germ cell cancers-International Germ Cell Cancer Collaborative Group. J Clin Oncol.1997;15: 594-603.

9. Motzer RJ, Sheinfeld J, Mazumdar M, Bains M, Mariani T, Bajic J, et al. Paclitaxel, ifosfamide, and cisplatin second-line therapy for patients with relapsed testicular germ cell cancer. J Clin Oncol. 2000;18: 2413-8.
10. Gilligan T, Beard C, Carneiro B, Chism D, Cost N, Derweesh IH, et al. NCCN Guidelines Version 2, 2018. Testicular Cancer

11. Feldman DR, Hu J, Dorff TB, Lim K, Patil S, Woo $\mathrm{KM}$, et al. Paclitaxel, ifosfamide, and cisplatin efficacy for first-line treatment of patients with intermediate or poor-risk germ cell tumors. J Clin Oncol.2016;34: 2478-83.

12. National Cancer Institute. Common Terminology Criteria for Adverse Events v4.0. http://ctep.cancer.gov/ protocolDevelopment/electronicapplications/ctc.htm

13. Kondagunta GV, Bacik J, Sheinfeld J, Bajorin D, Bains $M$, Reich $L$ et al. Paclitaxel plus ifosfamide followed by high-dose carboplatin plus etoposide in previously treated germ cell tumors. J Clin Oncol. 2007;25: 85-90.

14. Nakamura T, Miki T. Recent strategy for the management of advanced testicular cancer. Int J Urol. 2010;17: 148-57.

15. Kurobe M, Kawai K, Oikawa T, Ichioka D, Kandori S, Takaoka E. et al. Paclitaxel, ifosfamide, and cisplatin (TIP) as salvage therapy and consolidation chemotherapy for advanced germ cell tumor. J Cancer Res Clin Oncol. 2015;141: 127-33.

16. Mead GM, Cullen MH, Huddart R, Harper P, Rustin GSJ, Cook PA, et al. A phase II trial of TIP given as second line (post -BEP) salvage chemotheraphy for patients with metastatic germ cell cancer; a medical research council trial. Br J Cancer. 2005;93: 178-84.

17. Feldman DR, Bosl GJ, Sheinfeld J, Motzer RZ. Medical treatment of advanced testicular cancer. JAMA. 2008;299: 672-84. 